\title{
Length scale, quasiperiodicity, resonances, separatrix crossings, and chaos in the weakly relativistic Zakharov equations
}

\author{
G. I. de Oliveira and F. B. Rizzato \\ Instituto de Física, Universidade Federal do Rio Grande do Sul, Caixa Postal 15051,
} 91501-970 Porto Alegre, Rio Grande do Sul, Brazil

A. C.-L. Chian

National Institute for Space Research-Instituto Nacional de Pesquisas Espaciais, P.O. Box 515, 12227-010 São José dos Campos, São Paulo, Brazil

(Received 11 January 1995)

\begin{abstract}
Nonlinear saturation of unstable solutions to the weakly relativistic, one-dimensional Zakharov equations is considered in this paper. In order to perform the analysis, two quantities are introduced. One of them, $\rho_{*}$, is proportional to the initial energy of the high-frequency field, and the other is the basic wave vector of the low-frequency perturbing mode $k=2 \pi / L$, with $L$ as the length scale. With these quantities it becomes possible to identify a number of regions on a $\rho_{*}$ versus $k$ parametric plane. For very small values of $\rho_{*}$, steady-state solutions become unstable when $k$ is also very small. In this case ion-acoustic dynamics is found to be unimportant and the system is numerically shown to be approximately integrable, even if $k$ is below a critical value where the solutions are not simply periodic. For larger values of $\rho_{*}$ the unstable wave vectors also become larger and the ion-acoustic fluctuations turn into active dynamical modes of the system, driving a transition to chaos, which follows initial inverse pitchfork bifurcations. The transition includes resonant and quasiperiodic features; separatrix crossing phenomena are also found. The influence of relativistic terms on the chaotic dynamics is studied in the context of the Zakharov equations; it is shown that relativistic terms generally enhance the instabilities of the system, therefore anticipating the transition.
\end{abstract}

PACS number(s): 52.35.Ra, 47.20.Ky

\section{INTRODUCTION}

Langmuir turbulence has been one of the most studied problems in modern nonlinear plasma physics. Over the last few years a great deal of effort has been directed to the analysis of Langmuir turbulence and related subjects such as soliton dynamics, collapse, nucleation of cavitons, and electromagnetic emission [1-7]. Langmuir turbulence is governed by the Zakharov equations which nonlinearly couple the slowly varying amplitude of a high-frequency electrostatic field (the Langmuir field) to low-frequency density fluctuations (the ion-acoustic field). High frequencies are close to the plasma frequency and low frequencies are about the same order of magnitude as the normal mode frequency of the ion-acoustic field, the ionacoustic resonant frequency. When the shortest time scale characterizing the motion is much larger than the time scale associated with the ion-acoustic resonant frequency, one lies in the so called subsonic regime. The other limiting regime where the longest time scales are sufficiently smaller than the ion-acoustic resonant time scale is called supersonic. In modulational regimes, for instance [7], the presence of subsonic or supersonic dynamics depends on whether the ratio of the Langmuir field density over the plasma thermal density is smaller or greater than the ratio of the electron mass over the ion mass.

In the absence of dissipation, the governing set of equations in the subsonic regime may be approximated by a nonlinear Schrödinger equation (NLS) which is an inte- grable equation. What happens in this case is that the ion-acoustic field becomes enslaved to the Langmuir field, with the system turning out to be periodic or quasiperiodic. On the other hand, when the dominant frequency of the density fluctuations is not sufficiently small, ionacoustic degrees of freedom become active, destroying integrability and driving a transition to chaos. One aim of the present work is to identify a variety of regions in the appropriate parameter space where one can expect periodic, quasiperiodic, or chaotic behaviors, providing new results and some new perspectives on topics not fully explored.

In particular, we also incorporate relativistic effects in the analysis. As far as linear stability analysis is concerned, it has been shown that relativistic mass corrections of the high-frequency electronic motion play a relevant role in the dynamics of the system [8]. In fact, it is shown that these corrections, besides altering the location of instabilities on the parameter space, can also modify the related growth rates of the unstable modes. In the present paper we shall go further into the investigation of these aspects; we shall examine the influence of the relativistic terms on the saturation properties of the initially unstable states. It shall be seen that relativistic effects generally tend to enhance the instabilities of the system. In particular it shall be seen that these relativistic terms, even if perturbatively smaller than the others present in the governing equations, may be responsible for turning regular solutions into chaotic ones. In the face of the possibility of this drastic transformation, it is then concluded that relativity cannot be totally dis- 
carded as is sometimes done for this type of system. As we shall be mostly interested in relativistic regimes where pump amplitudes are relatively large, we do not include dissipative processes in the present analysis.

To perform our investigation, we first determine in which regions of the parameter space our system is integrable. Then one can have a good initial idea of where chaos is likely or unlikely to develop. This parameter space depends on the particular setting we wish to investigate and the appropriate setting for the instabilities we shall study here is defined as follows. One builds up a single stationary mode of the Langmuir field containing a certain amount of energy, and perturbs this equilibrium solution with a disturbance characterized by a lowfrequency wave vector $k$. The wave vector, in particular, sets the length scale of the problem. These two quantities, the initial energy (actually a quantity proportional to the initial energy) and the basic wave vector, define two axes of a bidimensional space which is thus taken as the sought-for parameter space.

Starting from the initial situation specified above and imposing some restrictions on the initial energy of the Langmuir field and on the wave vector of the perturbing mode, the Fourier series of the nonlinear developing solutions can be truncated to three terms. Two are the restrictions justifying the truncation procedure: (i) the possibility of enslaving the low-frequency dynamics to the high-frequency one, whereupon by disregarding time derivatives in the equation for density fluctuations one obtains the NLS mentioned above; and (ii) choosing the perturbing wave vector such that its harmonics $l k(l=2,3,4, \ldots)$ are outside the unstable band of the initial instabilities.

It is found that both restrictions are satisfied on a well defined domain of the parameter space. Within this domain the dynamical system is expected to be well described by a low-dimensional approximation of the NLS, with correspondingly simple and analytically integrable solutions. As one abandons the low-dimensional region, solutions are thus likely to lose their simplicity. It shall be seen that this is indeed the fact. With analytical estimates, numerical simulations, and the analysis of Lyapunov diverging trajectories we shall see that the outcoming irregular solutions can be of two types: (a) for very small values of the initial energy they are quasiperiodic with no associated chaos; (b) for larger values of the initial energy, solutions become chaotic with positive Lyapunov exponents if one is below a critical wave number or above a critical level of the relativistic perturbation.

We note that in case (a) the system is still well approximated by a NLS which, however, can no longer be truncated to a few Fourier modes. The nonchaotic region on the parameter space is the union of the low-dimensional and quasiperiodic regions.

Transition to chaos is examined in the large energy regimes as a function of the length scale. As one gradually goes into chaos one first observes resonant states on a 2-torus [2] along with a finite number of period doubling bifurcations. Going deeper into chaos, higherdimensional effects seem to prevent the creation of the 2- torus and the corresponding resonant states $[9,10]$. The transition follows an initial inverse pitchfork bifurcation which sets the system close to a heteroclinic trajectory involving one stable fixed point and two distinct unstable fixed points. States with larger number of unstable points are also found to exist as the low-frequency wave vector is varied $[11,12]$.

Finally, it is also shown that the relativistic-induced transition to chaos follows a similar route.

The paper is therefore organized as follows. In Sec. II we introduce the full set of equations and derive the lowdimensional approximation identifying its validity region on the appropriate parameter space; in Sec. III we perform various simulation runs in order to check the analytical estimates of Sec. II; in Sec. IV we analyze the typical transition to chaos; in Sec. V we investigate the role of relativistic effects; and in Sec. VI we conclude the work.

\section{GOVERNING EQUATIONS AND LOW-DIMENSIONAL DYNAMICS}

\section{A. Governing equations}

Let us first write down the normalized form of the weakly relativistic Zakharov equations which shall be used in the following analysis. These equations are [8]

$$
i \partial_{t} E+\partial_{x}^{2} E=n E-\alpha|E|^{2} E
$$

and

$$
\partial_{t}^{2} n-\partial_{x}^{2} n=\partial_{x}^{2}|E|^{2}
$$

where $\partial_{t} \equiv \partial / \partial t, \partial_{x} \equiv \partial / \partial x ; E=E(x, t)$ is the slowly varying amplitude of the electric field normalized to $8 \sqrt{\pi n_{0} m_{e} T_{e} /\left(3 m_{i}\right)}$ with $n_{0}$ as the equilibrium density, $T_{e}$ as the electron temperature, and $m_{e, i}$ as the electron (ion) mass; $n=n(x, t)$ are the low-frequency density fluctuations normalized to the equilibrium density, and $\alpha$ is the weakly relativistic parameter given by $\alpha \equiv 3 k_{B} T_{e} / m_{e} c^{2} \ll 1$ in the dipolar approximation, with $k_{B}$ as the Boltzmann constant and $c$ as the velocity of light. Time and coordinate are adimensionalized as $\left(m_{e} / m_{i}\right) \omega_{p} t \rightarrow t$ and $\sqrt{m_{e} / m_{i}}\left(\omega_{p} / v_{e}\right) x \rightarrow x$, respectively, where $\omega_{p}$ is the electron plasma frequency and $v_{e}^{2}=k_{B} T_{e} / m_{e}$.

We also assume the fields to be spatially periodic, with $L=2 \pi / k$ defining the basic length scale.

\section{B. Low-dimensional dynamics and its validity conditions}

The low-dimensional model can be obtained if one Fourier expands all the dynamical variables as

$$
Q(x, t)=\sum_{p=-\infty}^{+\infty} Q_{p}(t) e^{i p k x}
$$


where $Q$ is an arbitrary quantity, and makes use of the following assumptions. First one truncates the series for the Langmuir field to three modes,

$$
E(x, t)=E_{0}(t)+E_{1}(t) e^{i k x}+E_{-1}(t) e^{-i k x},
$$

noticing that the dipolar approximation $k_{0}=0, k<1$ is used to rule out the presence of induced higher harmonics of density fluctuations whose effects could be of the same order of magnitude as the relativistic ones.

Next one assumes that the time scale satisfies $\partial_{t}^{2} \ll \partial_{x}^{2}$ to get

$$
n=n_{1} e^{i k x}+n_{2} e^{2 i k x}+\text { c.c. }
$$

with

$$
n_{1}=-E_{1} E_{0}^{*}-E_{-1}^{*} E_{0} \quad \text { and } \quad n_{2}=-E_{1} E_{-1}^{*} .
$$

In view of the fact that for a real variable $n_{p}=n_{-p}^{*}$, these two relations are sufficient to determine the lowfrequency field.

On substituting relations $n_{1}$ and $n_{2}$ into Eqs. (1), writing

$$
\begin{aligned}
E_{0,1,-1}(t) \equiv & \sqrt{\rho_{0,1,-1}(t)} e^{i \phi_{0,1,-1}(t)} \\
& \times e^{\int^{t} \alpha\left(\left|E_{0}\right|^{2}+\left|E_{1}\right|^{2}+\left|E_{-1}\right|^{2}\right) d t},
\end{aligned}
$$

defining $\psi \equiv \phi_{1}+\phi_{-1}-2 \phi_{0}$, and separating the lowdimensional group of equations into real and imaginary parts, one arrives at the following equations for the variables $\rho_{0}$ and $\psi$ :

$$
d_{t} \rho_{0}=-4(1+\alpha) \rho_{0}\left(\rho_{*}-\rho_{0}\right) \sqrt{1-\cos ^{2} \psi}
$$

and

$$
\begin{aligned}
d_{t} \psi= & 2(1+\alpha)\left[-\frac{k^{2}}{1+\alpha}+\left(2 \rho_{0}-\rho_{*}\right) \cos \psi\right. \\
& \left.+\frac{1}{2}\left(3 \rho_{0}-\rho_{*}\right)\right]
\end{aligned}
$$

with

$$
\rho_{*} \equiv \rho_{0}(t=0)
$$

and $d_{t} \equiv d / d t$. These equations can be derived from the Hamiltonian

$$
\begin{aligned}
H= & (1+\alpha)\left[2 \rho_{0} \sqrt{\rho_{1} \rho_{-1}} \cos \psi-\frac{k^{2}}{1+\alpha}\left(\rho_{1}+\rho_{-1}\right)\right. \\
& \left.+\rho_{1} \rho_{-1}+\rho_{1} \rho_{0}+\rho_{-1} \rho_{0}\right]
\end{aligned}
$$

if one takes the $\rho_{j}$ 's and $\phi_{j}$ 's as, respectively, conjugated canonical momenta and coordinates, noticing that $\rho_{0}(t)+$ $2 \rho_{1,-1}(t)=M_{1,-1}$ where the $M$ 's are constants of motion with $M_{1}=M_{-1}=\rho_{*}$ to satisfy the initial conditions $\rho_{1,-1}(t=0) \approx 0.0$. In addition, the trajectory satisfying the initial conditions is the one with $H=0.0$.

The validity conditions for the various formulas obtained so far and the additional requirement of a nontrivial solution generate a set of restrictions on the parameters used above. These restrictions define a region in the parameter space where the low-dimensional approximation obtained above is expected to be accurate. We shall refer to this region as the low-dimensional region.

To determine the restrictions and the location of the low-dimensional region, let us first examine where some nontrivial dynamics take place. To this end we merely observe that saturation occurs when $1-\cos ^{2} \psi=0$. On substituting the expression for $\cos \psi$ obtainable from (9) and $M_{1}=M_{-1}=\rho_{*}$ into this equality one gets

$$
\left(\rho_{0}\right)_{s a t}=\left(\frac{k^{2}}{1+\alpha}-\frac{\rho_{*}}{4}\right) \frac{4}{7}
$$

at saturation. If one now demands $\left(\rho_{0}\right)_{s a t}<\rho_{*}$ to allow for some energy exchange between pump and daughter waves, one has to satisfy

$$
0<k<k_{s} \equiv \sqrt{\frac{2 \rho_{*}}{1+\alpha}},
$$

otherwise $\rho_{0}(t)$ would always be equal to $\rho_{*}$ and a nontrivial dynamics would not develop. The dependence of $k_{s}$ (and the other thresholds) on $\alpha$ and $\rho_{*}$ shall not be explicitly indicated unless necessary.

Use of Eqs. (7) and (8) or of Hamiltonian (9) shows that if $k_{s} / 2<k<k_{s}$ there are three fixed points located at $\rho_{0} \leq \rho_{*}$ and none at $\rho_{0}>\rho_{*}$. These points, which can be seen in Fig. 1(a), are labeled $(j=1,2,3)$, the first two being unstable and the last stable. Further use of the Hamiltonian (9) shows that these three points are created as a $k>k_{s}$ unstable fixed point with coordinates

$$
\rho_{0}=\frac{2}{7}\left(\frac{k^{2}}{1+\alpha}+\frac{3}{2} \rho_{*}\right) \quad \text { and } \quad \psi=0
$$

[see Fig. 1(b) where we take $\rho_{*}=0.2, \alpha=0$, and $k=0.7>k_{s}$ ] undergoes an inverse pitchfork bifurcation at $k=k_{s}, \rho_{0}=\rho_{*}, \psi=0$. We point out that the $\rho_{0}>\rho_{*}$ fixed point might be naively seen as spurious because it could be said to be associated with nonphysical states such as $\rho_{1,-1}<0$ as can be seen from Eqs. (9) and the respective conserved quantities. However, this is not true. The arguments above indicate that this point turns out to be of relevance in the analysis of the physical steady-state fixed points [note that the $\rho_{0}$ coordinate of the third fixed point for $k<k_{s}$ is also determined by expression (11)]. We shall refer to the region containing trapped orbits around the third fixed point as the first island.

If $k<k_{s} / 2$, Eqs. (7)-(9) indicate that a similar inverse pitchfork bifurcation creates a new triplet of fixed points whose elliptic point is located at $\psi=\pi$ and the hyperbolic points at $\rho_{*}=0$; we shall refer to the trapping region around the third elliptic fixed point as the second island. This is what can be seen in Fig. 1(c). The 
importance of this new triplet lies in the fact that for a convenient choice of initial conditions and for not too large energies, the resulting orbit can keep continuously hopping from one to another's attracting region, which in turn can create separatrix crossings and separatrix chaos $[11,12]$. We shall return to this point in Sec. IV.

Another validity condition is based on the assumed stability of perturbing modes with higher harmonics of the basic wave vector $k$. If higher wave vectors $l k(l=$ $\pm 2, \pm 3, \ldots$ ) become unstable, new dynamical fields of the form $E_{l}$ should also be included in the system, breaking down the low dimensionality of the model.
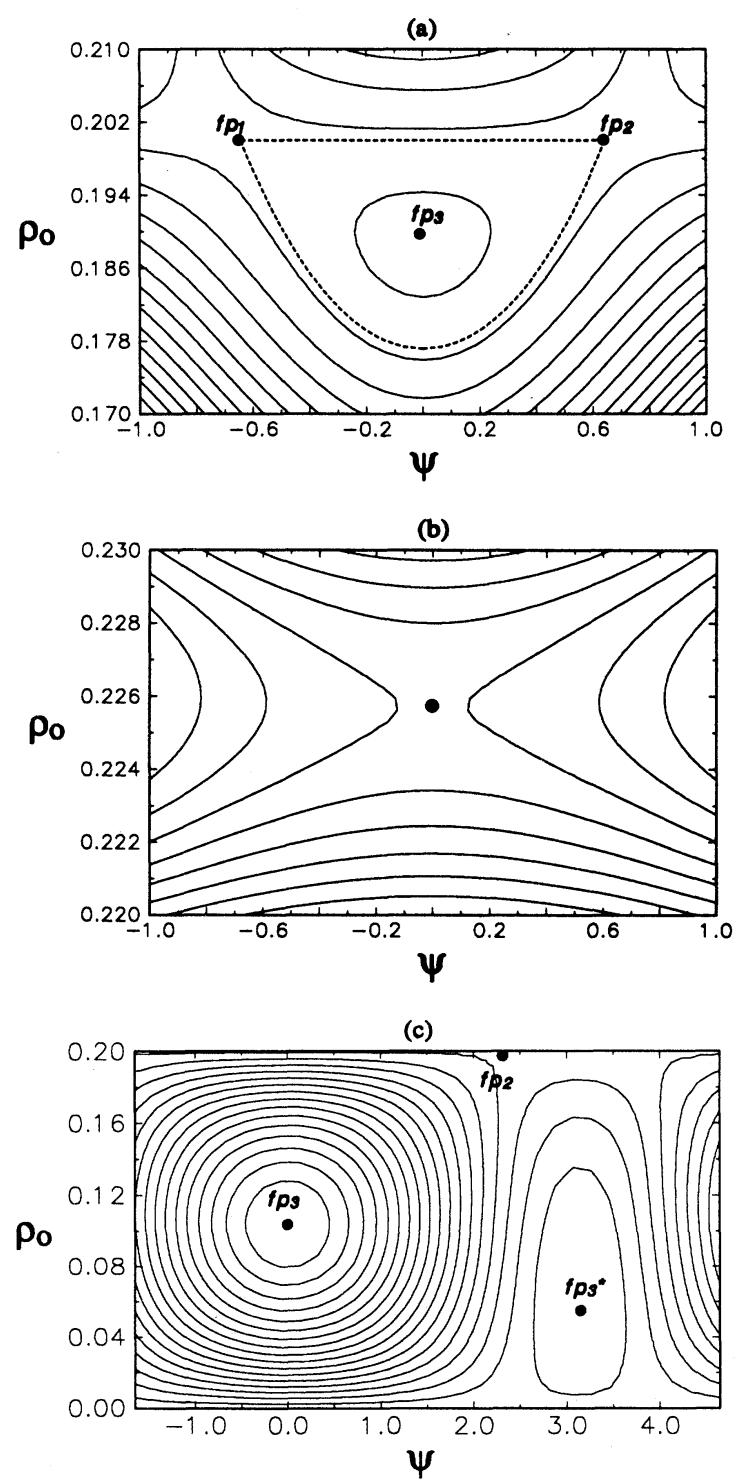

FIG. 1. Low-dimensional trajectories on the $\left(\rho_{0}, \psi\right)$ plane; $\rho_{*}=0.2$ with $k_{s} / 2<k=0.6<k_{s}$ in (a) and $k=0.7>k_{s}$ in (b). The fixed points are labeled. The dashed trajectory is the one satisfying the initial condition with $H=0.0$. In (c) the second island can be seen with $k=0.252<k_{s} / 2$.
The instability band for a given value of $\rho_{*}$ has been obtained above; it reads $0<k<k_{s}$. But now, if one is to avoid the destabilization of the next (second) harmonics, what one has to do is just to demand $2 k>k_{s}$. This effectively sets the higher harmonics outside the unstable band and implies

$$
k>k_{h} \equiv \frac{k_{s}}{2}=\sqrt{\frac{\rho_{*}}{2(1+\alpha)}},
$$

which is to be considered as our second validity condition.

The last condition can be obtained when one recalls that the ion-acoustic field was considered as being enslaved to the Langmuir one. In the modulational regime, which is the one operating here, this is equivalent to saying that the initial growth rate of the instability, $\gamma$, must be much smaller than the normalized ion-acoustic time scale $|k|$ [see Eq. (2)], i.e., $\gamma \ll k$. The maximum initial growth rate can be obtained from (7) and (8) as $\gamma_{\max } \sim(1+\alpha) \rho_{*}$, from which follows the last condition,

$$
k \gg k_{i} \equiv 2(1+\alpha) \rho_{*} .
$$

Putting together all the three conditions defining the nontrivial low-dimensional dynamics, one arrives at the relations

$$
k_{h}<k<k_{s}
$$

and

$$
k \gg k_{i}
$$

The low-dimensional region can be further extended if one improves the estimates noticing that the ion-acoustic dynamics is also enslaved if $k$ lies within the narrow band

$$
1 \gg \frac{k_{s}-k}{k_{s}}>0
$$

[note that Eq. (12) is still satisfied]. In this case the $\cos \psi$ term of Eq. (7) tends to unity, causing $\gamma \rightarrow 0$ even if $\rho_{*}$ is such that $\gamma_{\max } \sim k$. Alternatively we can see this narrow band as a close neighborhood of the region where the inverse pitchfork bifurcation takes place, recalling that for this kind of critical configuration the instability of unstable points is relatively weak [13].

The restrictions above are represented in Fig. 2 for the nonrelativistic case $\alpha=0$; relativistic effects shall be more consistently considered later. Two scales of $\rho_{*}$ are represented in Figs. 2(a) and 2(b). Besides the narrow band close to $k_{s}$ mentioned previously, it is thus seen that the inequalities (12) and (13) can be simultaneously satisfied only when $\rho_{*}$ is very small [Fig. 2(a)]. If this is the case, the system is expected to be trivially stable above $k_{s}$, simply periodic between $k_{s}$ and $k_{h}$ (this segment of the $k$ axis is inside the low-dimensional region), and multifrequency quasiperiodic below $k_{h}$. In accordance with what was previously said, this quasiperiodic kind of behavior may arise for $k<k_{h}$ in view of the destabilization of many other modes besides the basic one; chaos is not expected because of the integrable properties of the NLS.

Curve $k_{i}$ defines the line of transition between sub- 
(a)

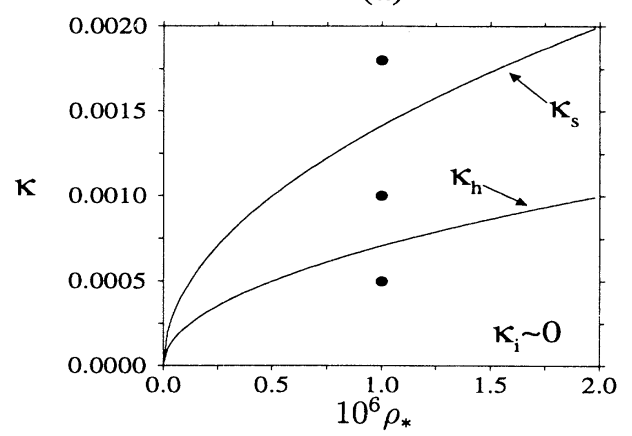

(b)

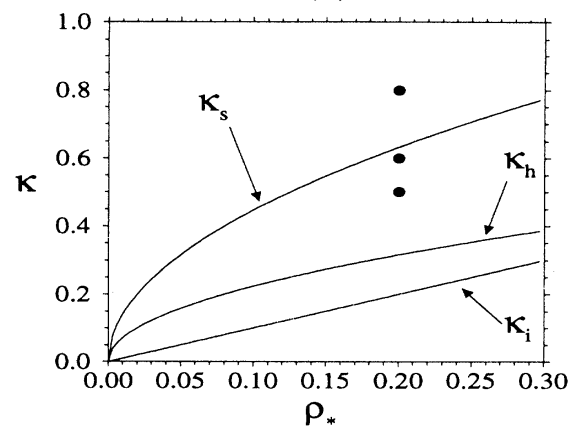

FIG. 2. Boundaries of the nonrelativistic $\alpha=0.0$ lowdimensional region. Small values of $\rho_{*}$ implying $k_{i} \ll k_{s, h}$ in (a) and larger values of $\rho_{*}$ implying $k_{i} \sim k_{s, h}$ in (b). Filled circles indicate points at which simulations are performed. sonic and supersonic ion-acoustic fluctuations. These ion-acoustic modes become dynamically and independently active if one is close to $k_{i}$. Their effects are thus effectively noticed only when $\rho_{*}$ is not excessively smaller than unity, a situation for which $k_{i}$ is not exceedingly small, as well. This is the case well represented by the scale adopted in Fig. 2(b).

Let us next proceed to check all the above assertions against numerical simulations based on the full set (1) and (2).

\section{INITIAL NUMERICAL SIMULATIONS}

\section{A. Numerical scheme}

In this section we shall perform a number of simulations to check the estimates obtained previously. As is somewhat usual for this kind of system, our simulation scheme consists of writing all the dynamical variables and the differential equations as Fourier series in the spatial variable. A number of modes $N$ ranging from $N=32$ to $N=128$ for each dynamical variable is used, nonlinear products in the differential equations are evaluated with a fast Fourier transform (FFT) subroutine, and the set of temporal equations is advanced in time with a predictorcorrector algorithm. Both the FFT and the predictorcorrector algorithm are subroutines of a Cray Y-MP2E computer. Numerical precision is tested by requiring stability against variation of tolerance factors and by monitoring the time evolution of the conserved quantity

$$
\mathcal{H}=\int_{0}^{L}\left[\left|\partial_{x} E\right|^{2}-\alpha|E|^{4}+n|E|^{2}+\frac{1}{2}\left(n^{2}+v^{2}\right)\right] d x
$$

with $\partial_{t} n=-\partial_{x} v$. In terms of $\mathcal{H}$, relative errors were found to be about one part in $10^{8}$ both in the nonrelativistic case and in the relativistic one to be studied later.

\section{B. Periodic, quasiperiodic, and chaotic regions}

Let us initiate the investigation by examining the behavior of temporal series for $\rho_{0}(t)$, considering very small values of $\rho_{*}, \rho_{*}=10^{-6}$. We present the results of the simulations in Fig. 3 for $k=1.8 \times 10^{-3}$ and $k=1.0 \times 10^{-3}$ [Fig. 3(a)] and $k=5.0 \times 10^{-4}$ [Fig. 3(b)]. Simulated points of the parameter space are indicated by filled circles in Fig. 2.

In the first case, whose representative point lies in the stable region of Fig. 2(a), above $k_{s}$, one has indeed a trivially stable solution. The second case corresponds to a point between $k_{s}$ and $k_{h}$ - in agreement with the estimates, one has a simply periodic solution. As already mentioned, the transition from $k<k_{s}$ to $k>k_{s}$ is accompanied by an inverse pitchfork bifurcation. In the third case one is below $k_{h}$ and the solution appears to be nonperiodic or irregular.

We now introduce a Lyapunov-like coefficient $\sigma_{L}$, defined as the average slope of the function $D(t)=$ $\ln [d(t) / d(t=0)]$ in a $D(t)$ versus $t$ plot, for relatively small values of $t$. The quantity $d(t)$ is the Euclidean distance between two initially close trajectories, calculated according to Doolen, Dubois, Rose, and Hafizi [2]. (a)

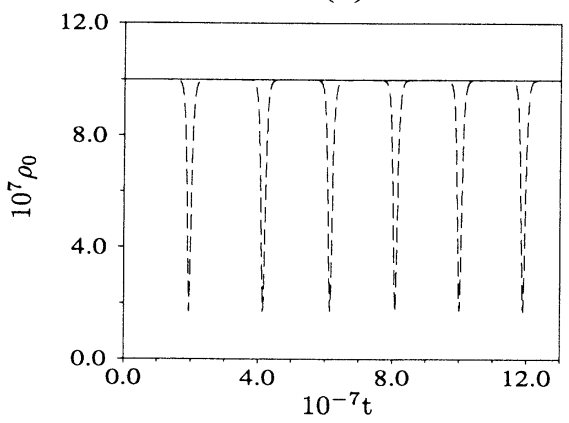

(b)

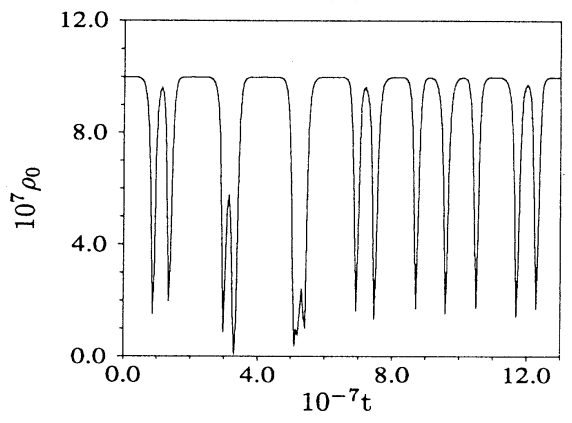

FIG. 3. Time series for $\rho_{0}(t)$ in the small $\rho_{*}$ regime, $\rho_{*}=10^{-6}$. Stable (solid line) and periodic (long dashed line) trajectories in (a), and quasiperiodic trajectory in (b). 
(a)

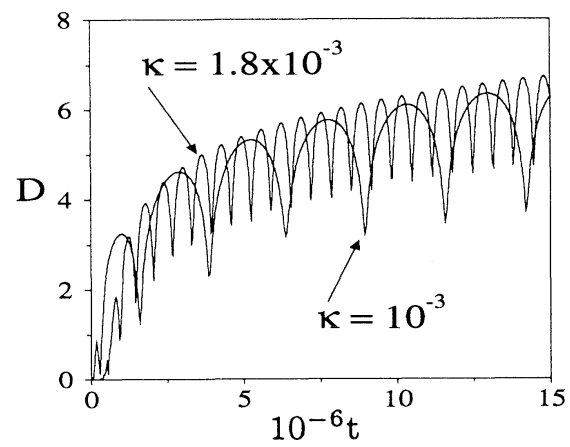

(b)

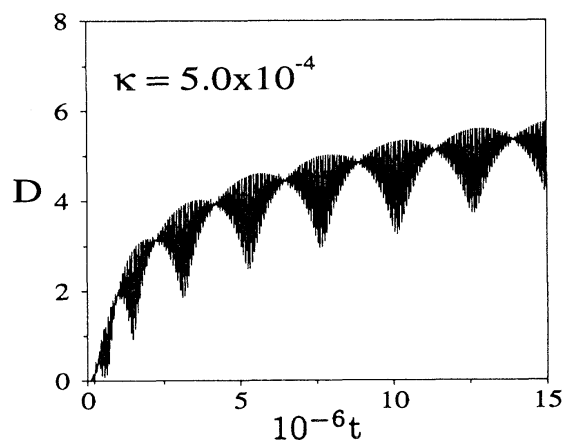

FIG. 4. The function $D(t)$ corresponding to the three cases of Fig. 3. The curves of (a) and (b) correspond to those of Figs. 3(a) and 3(b), respectively.
In Fig. 4 we plot $D(t)$ respectively corresponding to the three cases of Fig. 3. The plots show that in all cases the diverging trajectories go at most as $\ln (t)$, which signals the existence of integrable dynamics [14]. The irregular integrable trajectory occurring for $k=5.0 \times 10^{-4}$ is therefore a quasiperiodic one; as has been said, this is a feature to be expected in view of the properties of the NLS governing the system for such low values of $\rho_{*}$.

We now perform simulations corresponding to Fig. 2(b) in Figs. 5 (time series) and 6 (trajectory divergence), considering $\rho_{*}=0.2$.

For $k>k_{s}$ one has again a trivial stationary stable solution which is not shown in the figures. If $k$ is inside the narrow band close to $k_{s}$, as in Fig. 5 (a) with $k=0.62$, the dynamics is apparently periodic. However, the solution corresponding to $k_{h}<k=0.5<k_{s}$ [Fig. 5(b)] is chaotic. This is seen from Fig. 6(b) where a positive $\sigma_{L}$ can be clearly identified. Further reduction of $k$ causes no further alteration of the character of the chaotic solutions. Simulation runs show that for such values of $\rho_{*}$, once inside the chaotic region corresponding to a particular value of $\rho_{*}, \sigma_{L}$ does not heavily depend on $k$.

We finally point out that with the help of Fig. 7 it is possible to observe that although chaotic, the state characterized by the parameters of Figs. 5 and 6 involves a relatively small number of Fourier components of the dynamical variables. Recalling expansion (3), in the plot we represent $\operatorname{Re}\left\{n_{p}\right\}(t=250)$ versus $p$ to show that $p_{\text {max }}$ does not extend up to very large values; one typically has $p_{\max } \sim 1$. A gradual increase of the number of involved modes as $k$ goes deeper below $k_{h}$ is to be expected.

\section{TRANSITION TO CHAOS}

Knowing that there are regular and chaotic regions in the parameter space, it becomes relevant to analyze the possible routes to chaos present in the system. This issue has been partially addressed in Ref. [2]. By increasing the pump amplitude, the authors were able to observe the conversion of a predominantly simple periodic state into a two-frequency one. It was also observed that the newer frequency was smaller than the original and that just after the bifurcation the system would go into chaos. We intend to further pursue the matter in order to see how the transition evolves after the birth of the secund frequency.

To perform the analysis, in Fig. 8 we display a sequence of power spectra $\left|\rho_{0}(f)\right|^{2}$ with $\rho_{0}(f) \sim \int \rho_{0}(t) e^{i 2 \pi f t} d t$, considering the case $\rho_{*}=0.2$.

Starting in Fig. 8(a) with the regular case where $k=$ $0.62 \sim k_{s}$ (note that in this case the wave vector $k$ lies within the narrow band around $k_{s}$ ), it is seen that the spectrum is basically a discrete set of frequencies $l f_{1}$, $l= \pm 1, \pm 2, \ldots$, with $f_{1}$ characterizing the frequency of the associated simply periodic orbit. As one goes deeper below $k_{s}$, as in Figs. 8(b) and 8(c) [Fig. 8(c) is a magnification of $8(\mathrm{~b})]$ with $k=0.605065$, one can pinpoint some smaller frequencies, among which $f_{2}$ is the larger. This essentially two-frequency state is similar to the one detected in the simulations of Ref. [2].

Given that two frequencies control the overall dynamics in the vicinity of $k_{s}$, one concludes that the resulting (a)

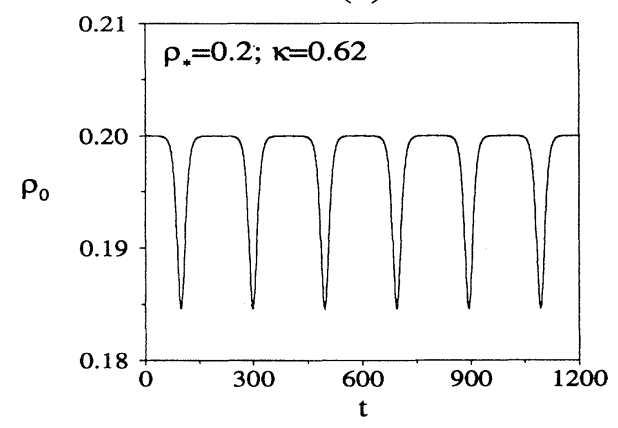

(b)

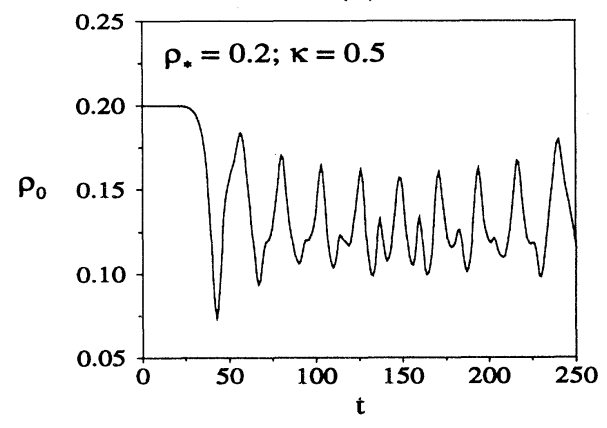

FIG. 5. Time series for $\rho_{0}(t)$ in the large $\rho_{*}$ regime, $\rho_{*}=0.2$. Periodic trajectory in (a) and chaotic trajectory in (b). 
(a)

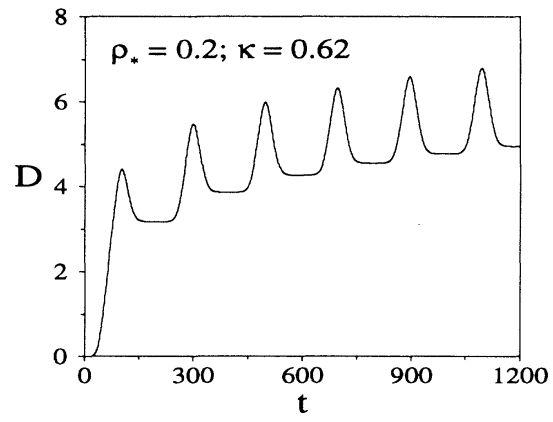

(b)

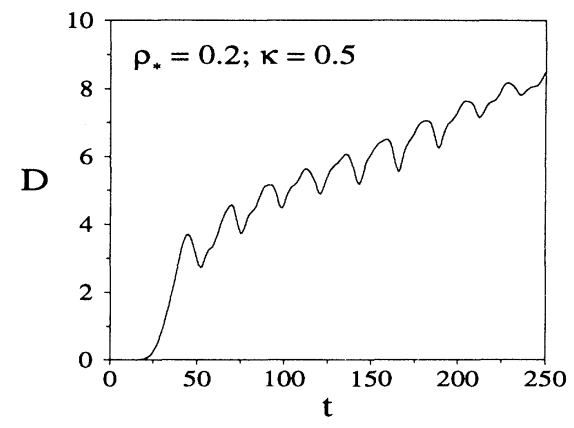

FIG. 6. The function $D(t)$ corresponding to the two cases of Fig. 5, respectively. trajectory develops close to a 2-torus whose axes are labeled by $f_{1}$ and $f_{2}$ (a similar phenomenon has been seen to occur with the NLS, as reported in Ref. [4]).

Then one continues to lower the wave vector $k$, as in Fig. $8(\mathrm{~d})$ where one considers $k=0.605$, to show a slightly chaotic state spreading along the horizontal axis. With the wave vector further diminished, one returns to what seems to be a predominantly nonchaotic state. This can be seen in Fig. 8(e) with $k=0.603$ where the power spectrum, at least momentarily, turns back into a discrete set of frequencies. For smaller values of $k, k=0.600$ [Fig. 8(f)], chaos sets in again.

We point out that this chaotic burst of Fig. 8(d) occurs just after a point where one can distinguish the following resonance condition involving $f_{2}$ and $f_{1}$ :

$$
j f_{2}=f_{1}
$$

with $j=13$ as seen from Fig. $8(\mathrm{~g})$. Some small chaotic activity is naturally expected as the dynamical trajectory is driven into a resonant state involving $f_{1}$ and $f_{2}$ by varying some control parameters. Further variation of the control parameters can drive the system off resonance, which explains the return to periodicity. One should not be surprised by this apparent coexistence of regular and chaotic regions; while this does not generically occur in dissipative systems, it is usual for conservative or Hamiltonian ones like ours.

We have also pursued period doubling bifurcations leading to chaos of frequency $f_{2}$, as would be likely to happen were resonant mechanisms really operative. We have indeed observed that $f_{2}$ undergoes period doubling while $13 f_{2}=f_{1}$, as can be seen in Figs. $8(\mathrm{~h})$ and $8(\mathrm{~g})$; the peak located at $f_{2} / 2$ is formed as $f_{3}$ and $f_{2}-f_{3}$ of Fig. 8(c) collapse against each other.

There is nothing extraordinary with the appearance of a half harmonic of a frequency $f$ in a power spectrum. What is important to be noted here is that just after the period doubling, frequency $f_{2}$ vanishes and the predominantly discrete spectrum is replaced by a noisy continuum. This is expected if a resonance takes place; as mentioned above, the period doubling route to chaos is a typical way to break resonant states. We did not detect higher-order period doubling of $f_{2}$ on its way to chaos. A more careful simulation may be able to observe this unless the period doubling cascade gets blurred due to higher-dimensional effects, as in the case studied by Ghosh and Papadopoulos [16]. In fact, the failure of our search for higher-order period doublings along with the fact that no period doubling of $f_{1}$ has also been detected may be an indication that resonant processes have indeed a finite operational range. Before going into this subject, note that the rate $f_{1} / f_{2}=13$ is exactly the one found in Ref. [2].

Bifurcations have not been seen for smaller values of $j$, such as, for example, for $j=2$ [see Eq. (16)] which would characterize the final period doubling cascade typical of low-dimensional resonant systems [15]. What happens is that too large values of $f_{2}$ do occur when $\left|k-k_{s}\right| / k_{s}$ is too large, as well. Then the mutual interaction of frequencies $f_{1}$ and $f_{2}$ with the set of ion-acoustic resonant frequencies seems to prevent the creation of a single 2-torus.

The low-dimensional point of view we have been using above is meaningful only when the trajectories of the system remain close to the first and second fixed points seen in Fig. 1(a) and their associated manifolds; the system would describe a close trajectory to a heteroclinic one determined by the low-dimensional set of variables. This is what happens for the parameters of Fig. 9(a), for instance. In this situation, in particular, the second island is far from being created because $k \sim k_{s}$ (recall the conditions for the creation of the second island).

The same sort of simulation can be performed for

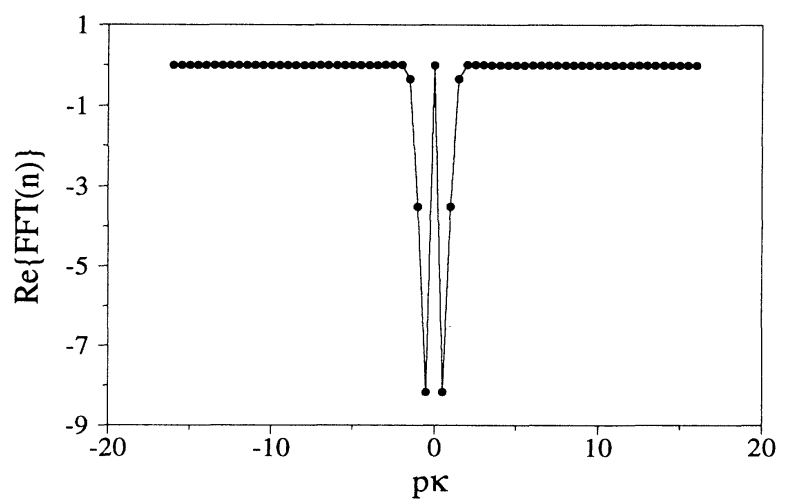

FIG. 7. Fourier spectrum for the density, $\operatorname{Re}\left\{n_{p}\right\}$, obtained at $t=250$. Few modes are involved in the dynamics. 
smaller values of $k$ as in Fig. 9(b) with $k=0.5$. In this regime the low-dimensional system could be viewed as losing an appreciable amount of energy to other degrees of freedom, especially to the degrees of freedom of the ion-acoustic field, dissipatively spiraling towards the stable fixed point (No. 3) which would be acting as a sink. Low-dimensional approximations would be inaccurate to represent this situation.

Both situations above refer to the case where the second island has not been created yet. Let us quickly examine what happens when the second island is created.

One possible situation is the one where the ion-acoustic wave is not strong enough to push the orbit away from the separatrix. In this case separatrix crossing turns out (a)

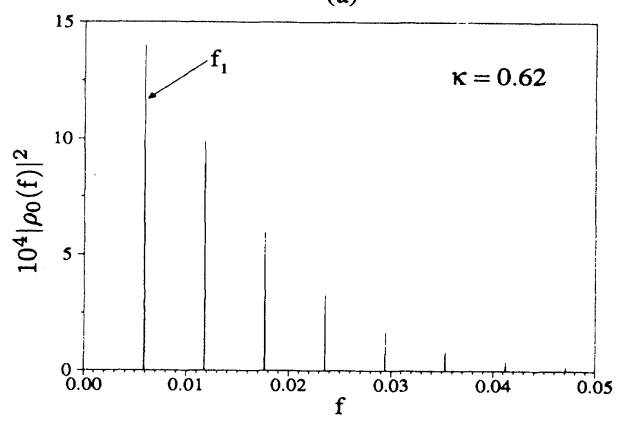

(c)

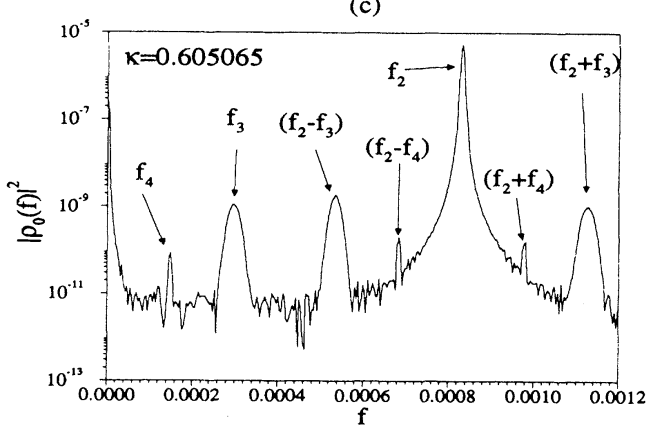

(e)

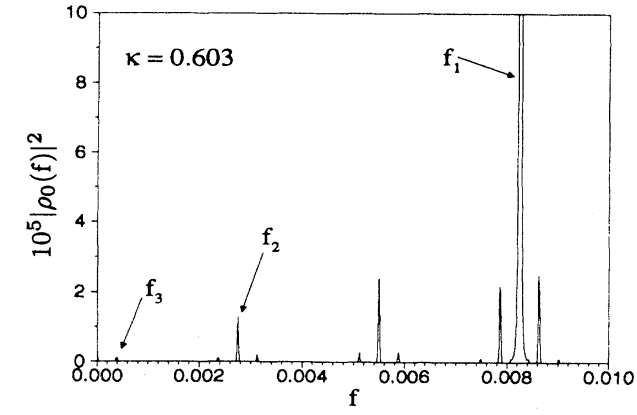

(g)

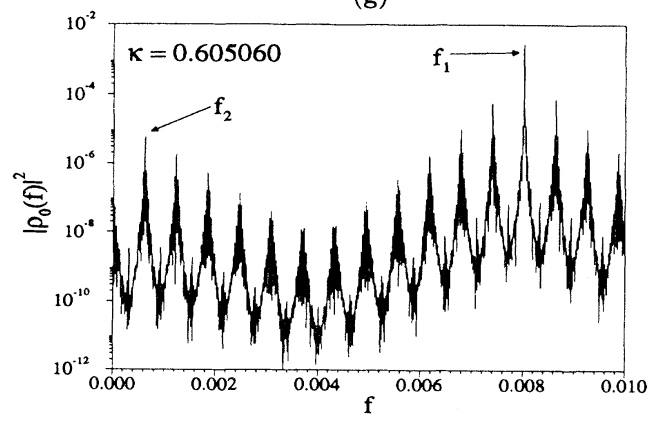

(b)

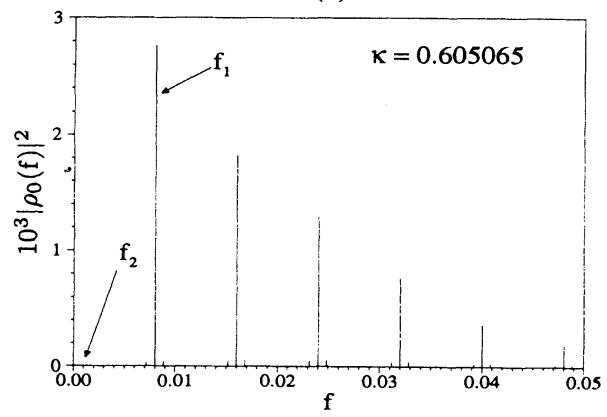

(d)

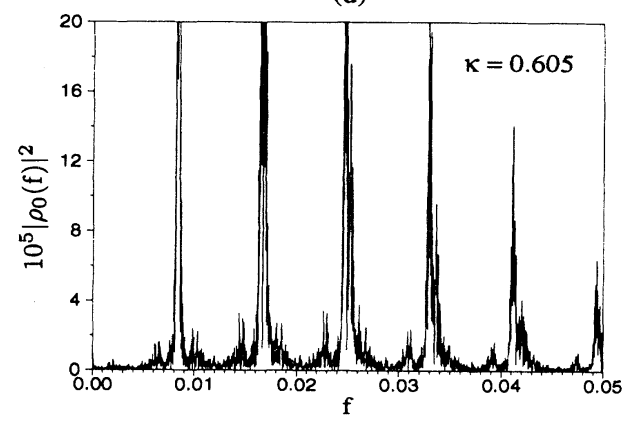

(f)

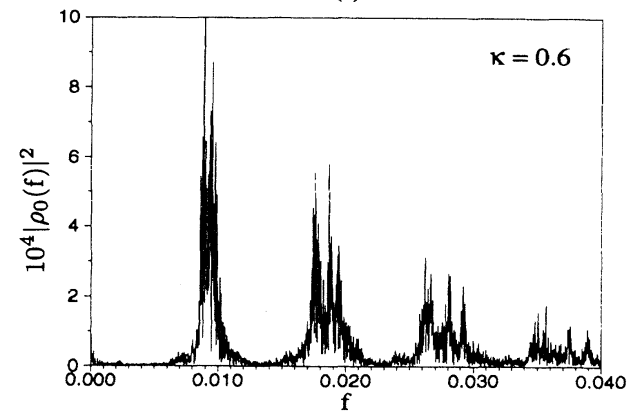

(h)

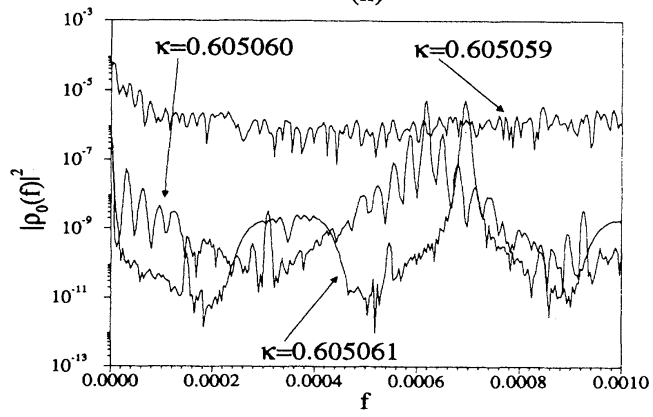

FIG. 8. Power spectra $\left|\rho_{0}(f)\right|^{2}$ as a function of decreasing values of $k$, with $\rho_{*}=0.2$ and $\alpha=0.0$. A predominantly periodic trajectory is analyzed in (a), quasiperiodic in (b) and (c), slightly chaotic in (d), quasiperiodic in (e), and chaotic in (f). Nonlinear resonance is displayed in $(\mathrm{g})$ and period doubling of $f_{2}$ leading to the slightly chaotic state of (d) in (h). 
to be an important ingredient in the chaotic dynamics. This can only happen for smaller values of $\rho_{*}$ than the one we have used here, $\rho_{*}=0.2$. Only then do the ionacoustic waves not drain a substantial amount of energy from the low-dimensional system, preventing the trajectory from remaining near the separatrix orbit and from executing the jumpings between the islands. In Fig. 9(c) we show the phase plane for $k>k_{s} / 2$, before the creation of the second island, and in Fig. 9(d) for $k<k_{s} / 2$, after the creation. Note that as the second island is created, the latter case, the orbit keeps jumping from one island to the other, thus filling the entire phase plane; before the creation, the orbit is noticeably constrained along the $\psi$ axis. For completeness we display the case $\rho_{*}=0.2$ in Fig. 9(e) with $k<k_{s} / 2$. Note that not even in this situation, where the second island is present, can separatrix crossings be observed. We emphasize that this occurs in view of the dissipative orbital spiraling towards elliptic fixed points (No. 3 in the present case) that does occur when the ion-acoustic wave is too strong, a feature associated with larger values of $\rho_{*}$. In all cases we have prepared initial conditions with $\psi=0$ and $\rho \sim \rho_{*}$.

In short, it seems that only if $k \sim k_{s}$ [Fig. 9(a)] or if $k_{s} / 2<k<k_{s}$ with $\rho_{*} \ll 1$, can the dynamics be well described by a possible low-dimensional approximation.

One can have chaos even in the absence of separatrix crossings, as in the situation depicted in Figs. 9(b) and $9(c)$. But if the second island is created and crossings become possible, as in Fig. 9(d), we expect some contribution to the chaotic dynamics due to resulting irregular separatrix crossings as analyzed by $[11,12]$.

We conclude this section with a final word on the two types of quasiperiodic behaviors occurring in the system. Quasiperiodicity may be present in both regimes (a)

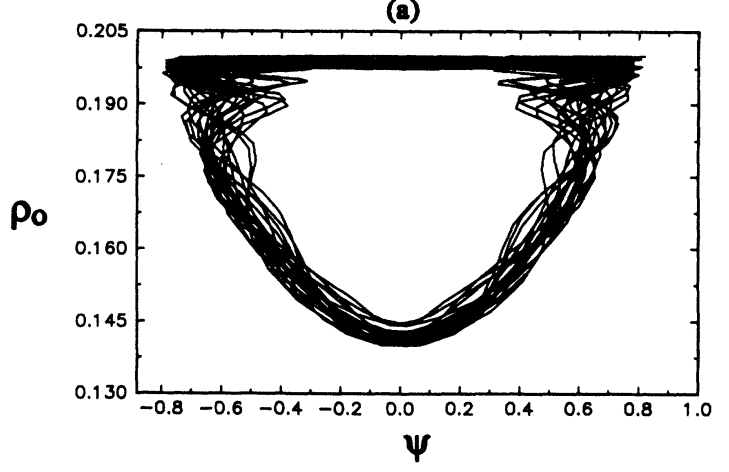

(c)

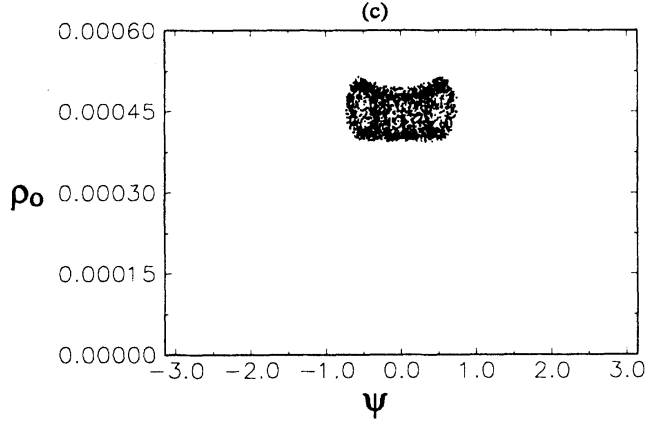

(b)
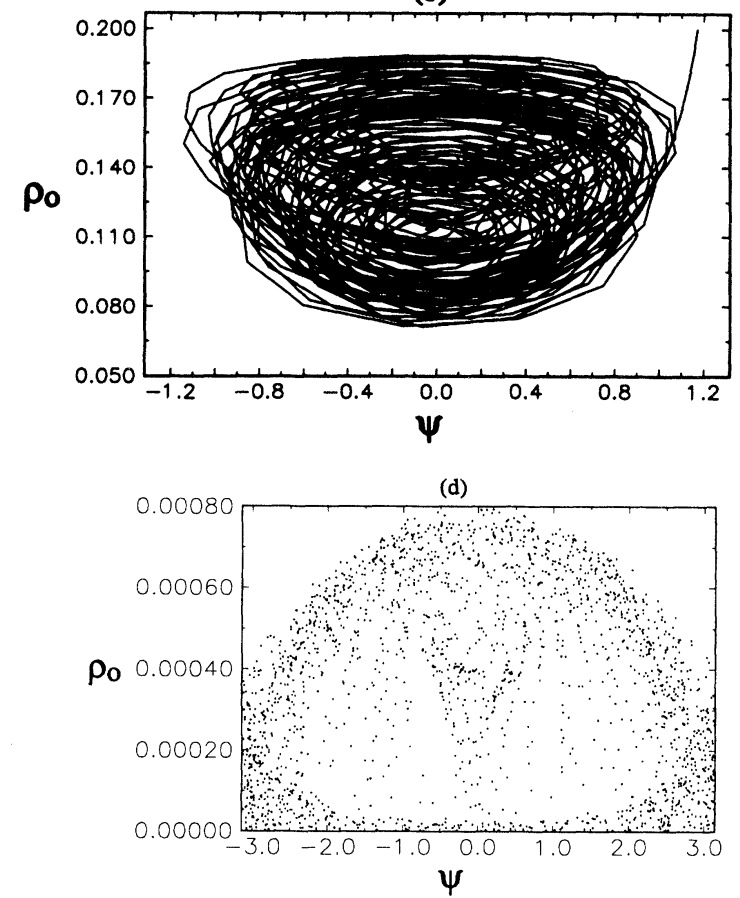

(e)

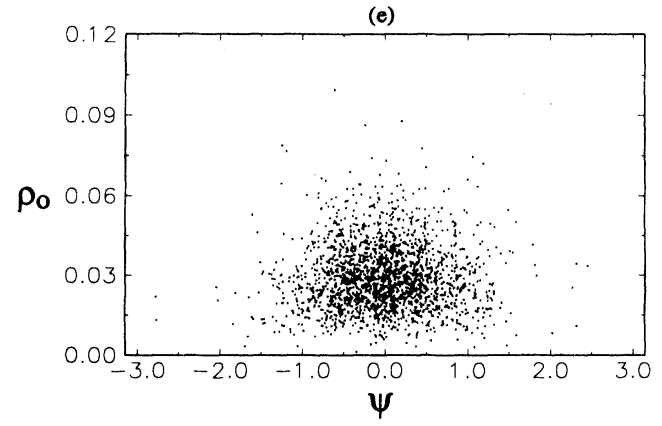

FIG. 9. Projection of the actual orbit on the $\left(\rho_{0}, \psi\right)$ plane with $\alpha=0.0$. In (a) $\rho_{*}=0.2$ and $k_{s} / 2<k=0.58$, in (b) $\rho_{*}=0.2$ and $k_{s} / 2<k=0.5$, in (c) $\rho_{*}=0.001$ and $k_{s} / 2<k=0.02981$, in (d) $\rho_{*}=0.001$ and $k_{s} / 2>k=0.01789$, and in (e) $\rho_{*}=0.2$ and $k_{s}>k=0.2529$. 
$k_{i} \ll k_{s, h}$ [Fig. 2(a)] and $k_{i} \sim k_{s, h}$ [Fig. 2(b)]. In the first case one has a broadband quasiperiodicity along the $k$ axis of the parameter space, generated by the destabilization of additional modes of the Langmuir field besides modes $E_{0,1,-1}$. In the second case quasiperiodicity is either due to low-dimensional orbits lying on a 2-torus (when $k$ is within a narrow band around $k_{s}$ ) or due to the mutual interaction of the low-dimensional frequencies and the ion-acoustic one (when $k$ is substantially smaller than $k_{s}$ ). We remark that only in the two last cases is quasiperiodicity involved with nonintegrable dynamics.

\section{RELATIVISTIC EFFECTS}

We finally analyze the weakly relativistic effects originated by the inclusion of mass variation in the highfrequency electronic dynamics. We start with the analytical estimate of Fig. 10 where we compare the positioning of curves $k_{s}, k_{h}$, and $k_{i}$ on the parameter space for the nonrelativistic and weakly relativistic cases, $\alpha=0.0$ and $\alpha=0.3$, respectively. It is immediately seen that relativistic terms tend to lower the instability thresholds of the system by displacing curve $k_{s}$ upwards. This has the important consequence that a regular low-dimensional state can be converted into a chaotic one by the inclusion of small relativistic effects.

Within the low-dimensional region of the parameter space, Eq. (7) shows that a weakly relativistic system with $\alpha \neq 0$ is equivalent to a nonrelativistic system with time and wave vector, respectively, rescaled as

$$
t_{\text {scaled }}=t(1+\alpha) \text { and } k_{\text {scaled }}=\frac{k}{\sqrt{1+\alpha}} .
$$

The relations above, in particular, allow one to compare the maximum initial growth rates in nonrelativistic and relativistic cases. The result is

$$
\left(\gamma_{\alpha}\right)_{\max }=(1+\alpha)\left(\gamma_{\alpha=0}\right)_{\max }=(1+\alpha) \rho_{*},
$$

which was derived earlier. Relations (17) and (18) and

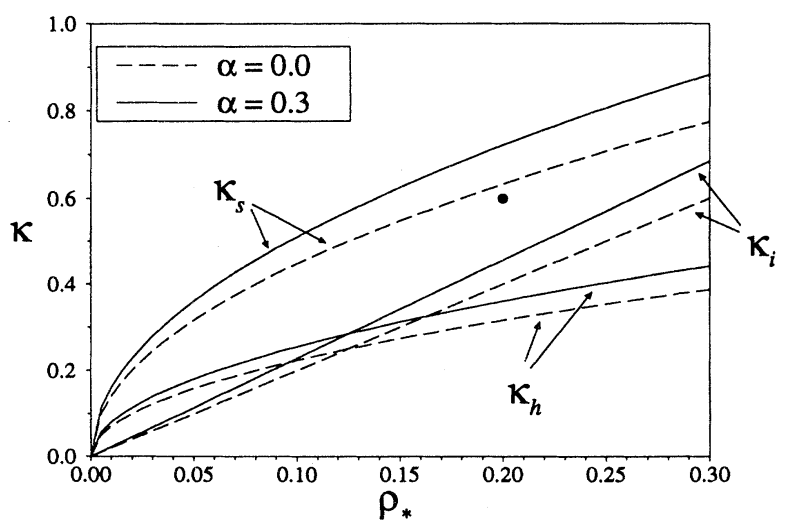

FIG. 10. Boundaries of the low-dimensional region with relativistic effects (full lines) included. Filled circle indicates the simulated point. the general aspect of Fig. 10 indicate that the basic role of relativistic effects on the regular dynamics is to enhance instabilities of the system. They also indicate that the increase of the relativistic factor $\alpha$ is equivalent to a decrease of the value of the wave vector $k$.

If one is considering large enough values of $\rho_{*}$ that chaos may be present in the system (depending on the choice of the wave vector), one could conjecture, in view of the above, that relativistic effects might induce chaos merely by bringing a stable or quasiperiodic point of the type $\left(\alpha=0, k \sim k_{s, \alpha}\right)$ into the unstable region $(\alpha>$ $\left.0, k<k_{s, \alpha}\right) \sim\left(\alpha=0, k_{\text {scaled }}<k_{s, \alpha=0}\right)$.

To check this hypothesis, in Fig. 11 we compare $D(t)$ for the nonrelativistic and weakly relativistic cases $\alpha=0$ and $\alpha=0.3$. We chose $\rho_{*}=0.2$ and a value for $k$ so close to $k_{s}$ (i.e., within the narrow band around $k_{s}$ ), $k=0.62$, that one can expect an integrable orbit developing for $\alpha=0$; the point where simulation was performed is represented by the filled circle of Fig. 10. Figure 11 indeed confirms the estimate, revealing a $D(t)$ behaving as a $D(t) \sim \ln (t)$. Now, as the weakly relativistic case $\alpha=0.3$ is considered, one can clearly see that the integrable orbit is converted into a chaotic one with a positive $\sigma_{L}$. Note that the weakly relativistic Zakharov equations are formally correct only under the assumption of very small values of $\alpha$. In practice, however, larger values can be used. We chose this slightly larger (but still small) value in Fig. 11 in order to provide a clear illustration of the effect. Much smaller values do appear in the analysis of transition to chaos, next.

On the whole, one concludes that under certain circumstances small relativistic corrections can really cause profound distortions of the physics of the relevant saturating solutions. Because of the equivalence between $k$ and $\alpha$ one would also infer that if $\alpha$ were used as a bifurcation control parameter, the route to chaos would be similar to the one associated with variations of $k$. All of this proves to be true. In fact, the sequenced power spectra displayed in Fig. 12 as function of increasing $\alpha$ reveal similar behavior to the one of Fig. 8. Note that a resonant regime again appears to coincide with the chaotic activity of Fig. 12(b).

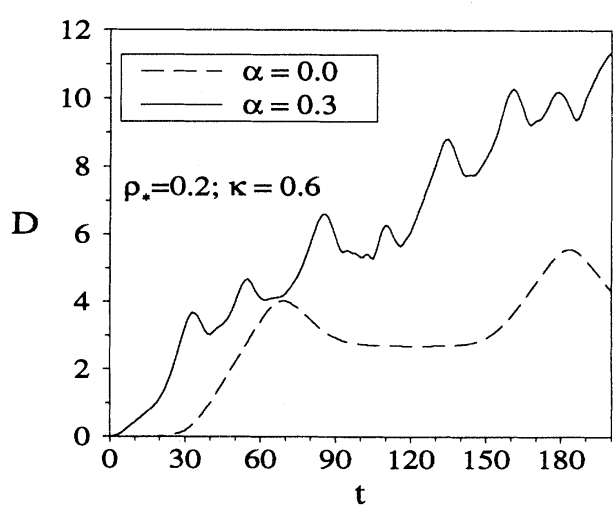

FIG. 11. The function $D(t)$ for the nonrelativistic (dashed line) and relativistic (full line) cases of Fig. $11 ; \rho_{*}=0.2$ and $k=0.62$. 
(a)

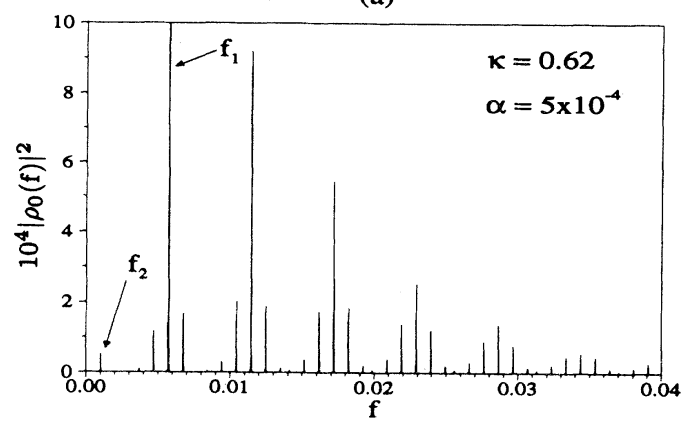

(b)

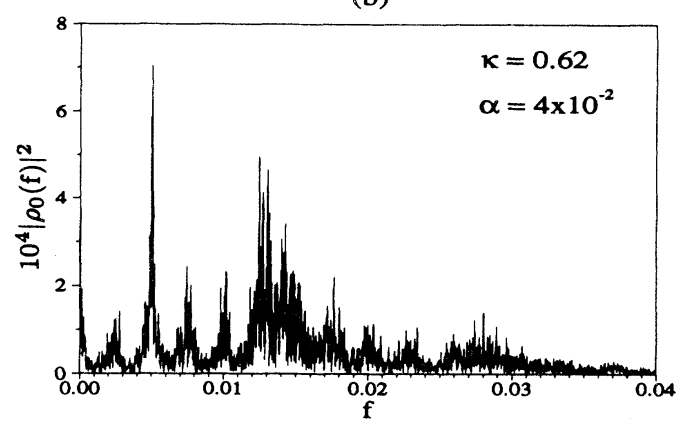

FIG. 12. Power spectra $\left|\rho_{0}(f)\right|^{2}$ as function of increasing values of $\alpha$ for $\rho_{*}=0.2$ and $k=0.62$.

\section{FINAL REMARKS}

In this paper we have considered the analysis of regular and chaotic dynamics associated with the weakly relativistic Zakharov equations.

A certain amount of energy was deposited on a steadystate mode which was rendered unstable through an inverse pitchfork bifurcation, and the nonlinear saturation of the initial instability was studied.

To begin with, we have located a certain region in the appropriate parameter space where the dynamics was expected to be low dimensional and regular. The regular dynamics within this region, the irregular dynamics (quasiperiodic and chaotic) outside the region, and the route to chaos were the subject of various simulation runs.

Transition to chaos as a function of the length scale of the system was found to involve resonant states with some of the corresponding period doubling bifurcations, and quasiperiodicity. The resonant and period doubled states appear to couple the principal frequencies of a 2torus and the last quasiperiodic stage couples these frequencies to the resonant ion-acoustic ones. During the initial phase of the transition, the trajectory remains close to a heteroclinic trajectory created by the inverse pitchfork bifurcation. Then, as one goes deeper into chaos, one observes two types of behaviors. (i) If $\rho_{*}$ is not too large, ion-acoustic waves are weakly nonlinear and cannot absorb a large quantity of the energy initially contained in the low-dimensional system. In this case, the trajectories remain close to the separatrix and the possibility of separatrix crossings does exist. In this case the chaotic character of the system may depend on the length scale because as $k$ is lowered past $k_{s} / 2$ chaotic contributions due to the separatrix crossings become relevant $[11,12]$. (ii) For larger values of $\rho_{*}$ ion-acoustic waves are fully nonlinear and can absorb a substantial amount of energy from the low-dimensional system. In this case the trajectories dissipatively spiral towards the central elliptic fixed points, diminishing the possibility of separatrix crossings even when the second island is created. In this situation the chaotic character of the system is independent of the length scale because of the absence of separatrix crossings as $k$ is lowered.

We have finally studied the influence of relativistic mass correction terms on the high-frequency electronic dynamics. With the help of plots, scaling rules, and numerical runs, it has been shown that relativistic terms enhance the unstable and chaotic features of the system. In particular we have shown that the inclusion of relativistic mass correction terms can convert regular orbits into chaotic ones through a quasiperiodic type of transition.

\section{ACKNOWLEDGMENTS}

The authors wish to thank Arthur Lopes and Iberê L. Caldas for helpful and interesting discussions. This work was partially supported by Financiadora de Estudos e Projetos (FINEP), Conselho Nacional de Desenvolvimento Científico e Tecnológico (CNPq), and Fundação de Amparo à Pesquisa do Estado de São Paulo (FAPESP), Brazil. Numerical computing was performed on the Cray Y-MP2E at the Universidade Federal do Rio Grande do Sul Supercomputing Center.
[1] S.G. Thornhill and D. ter Haar, Phys. Rep. 43, 43 (1978).

[2] G.D. Doolen, D.F. Dubois, H.A. Rose, and B. Hafizi, Phys. Rev. Lett. 51, 335 (1983).

[3] G.D. Doolen, D.F. Dubois, and H.A. Rose, Phys. Rev. Lett. 54, 804 (1985).

[4] H.T. Moon and M.V. Goldman, Phys. Rev. Lett. 53, 1821 (1984).
[5] P.A. Robinson and D.L. Newman, Phys. Fluids B 1, 2319 (1989).

[6] F.B. Rizzato and A.C.-L. Chian, J. Plasma Phys. 48, 71 (1992).

[7] A.C.-L. Chian and F.B. Rizzato, J. Plasma Phys. 51, 61 (1994).

[8] V.I. Berezhiani, N.L. Tsintsadze, and D.D. Tskhakaya, 
J. Plasma Phys. 24, 15 (1981).

[9] J. Newhouse, D. Ruelle, and F. Takens, Commun. Math. Phys. 64, 35 (1978).

[10] C. Grebogi, E. Ott, and J. Yorke, Physica D 15, 354 (1985).

[11] H.T. Moon, Phys. Rev. Lett. 64, 412 (1990).

[12] C. Zhou, X.T. He, and S. Chen, Phys. Rev. A 46, 2277 (1992).

[13] G. Corso and F.B. Rizzato, Physica D 80, 296 (1995).
[14] A.J. Lichtenberg and M.A. Lieberman, Regular and Stochastic Motion (Springer, New York, 1983).

[15] R. Pakter, G. Corso, T.S. Caetano, D. Dillenburg, and F.B. Rizzato, Phys. Plasmas 1, 4099 (1994); R. Pakter, R.S. Schneider, and F.B. Rizzato, Phys. Rev. E 49, 1594 (1994).

[16] S. Ghosh and K. Papadopoulos, Phys. Fluids 30, 1371 (1987). 